\title{
Electronic and Magnetic Structure of Possible Iron Based Superconductor $\mathrm{BaFe}_{2} \mathrm{Se}_{3}$
}

\author{
${ }^{a}$ M. V. Medvedev, ${ }^{b}$ I. A. Nekrasov, ${ }^{a, b}$ M. V. Sadovskii ${ }^{1)}$ \\ ${ }^{a}$ Institute for Electrophysics, Russian Academy of Sciences, Ural Branch, Amundsen str. 106, Ekaterinburg, 620016, Russia \\ ${ }^{b}$ Institute for Metal Physics, Russian Academy of Sciences, Ural Branch, S.Kovalevskoi str. 18, Ekaterinburg, 620990, Russia
}

Submitted December 2011

\begin{abstract}
We present results of LDA calculations (band structure, densities of states, Fermi surfaces) for possible iron based superconductor $\mathrm{BaFe}_{2} \mathrm{Se}_{3}$ (Ba123) in normal (paramagnetic) phase. Results are briefly compared with similar data on prototype $\mathrm{BaFe}_{2} \mathrm{As}_{2}$ and $(\mathrm{K}, \mathrm{Cs}) \mathrm{Fe}_{2} \mathrm{Se}_{2}$ superconductors. Without doping this system is antiferromagnetic with $T_{N}^{\exp } \sim 250 \mathrm{~K}$ and rather complicated magnetic structure. Neutron diffraction experiments indicated the possibility of two possible spin structures (antiferromagnetically ordered "plaquettes" or "zigzags"), indistinguishable by neutron scattering. Using LSDA calculated exchange parameters we estimate Neel temperatures for both spin structures within the molecular field approximation and show $\tau_{1}$ ("plaquettes") spin configuration to be more favorable than $\tau_{2}$ ("zigzags").
\end{abstract}

'PACS: 71.20.-b, 74.70.-b, 75.10.-b, 75.25.+z

\section{INTRODUCTION}

During the recent years novel iron based hightemperature superconductors [1] attracted a huge number of both experimental and theoretical investigations (for review see [2,3]). This flow of papers is still continuing uninterrupted. Major chemical classes of iron based 'superconductors are pnictides [1] and chalcogenides [4] '(for comparative study see Ref. [5]).

Recently $\mathrm{BaFe}_{2} \mathrm{Se}_{3}$ (Ba123) system [6] was proposed as possible superconductor (analogous to (K,Cs) $\mathrm{Fe}_{2} \mathrm{Se}_{2}$ ) 'with experimental indications of $T_{c} \sim 11 \mathrm{~K}$. However, later study claimed no superconductivity in this system down to $1.8 \mathrm{~K}$ [7]. Both papers [6, 7] exploited neutron diffraction and discovered Ba123 to be antiferromagnetic spin-ladder system with Neel temperature of 'about $250 \mathrm{~K}$, and proposed two possible spin configurations. In Ref. [6] these spin configurations (antifer'romagnetically ordered "plaquettes" or "zigzags") were reported to be equivalent in a sense of diffraction picture and thus indistinguishable. Ref. [7] is rather in favour of "plaquettes" pattern.

Most recent investigations of the Ba123 system shown that with doping (Fe deficiency) it becomes a semiconductor with the energy gap about $0.2 \mathrm{eV}$ at room temperature [8, 9]. In Ref. [9] LSDA calculations were also used to calculate total energies of phases with different magnetic structures. From these results it can be seen that more favorable is apparently the "plaquettes" configuration.

\footnotetext{
1)E-mail: sadovski@iep.uran.ru
}

In this work we present LDA calculated electronic structure, densities of states and Fermi surfaces for Ba123 system in (high-temperature) paramagnetic phase (probably most promising for superconductivity) and compare the results with those for the previously studied systems, such as $\mathrm{BaFe}_{2} \mathrm{As}_{2}$ and (K, $\left.\mathrm{Cs}\right) \mathrm{Fe}_{2} \mathrm{Se}_{2}$. For antiferromagnetic phase we use LSDA calculated values of Heisenberg model exchange parameters to calculate Neel temperatures for "plaquettes" and "zigzags" spin structures, to compare relative stability of both structures. To this end we conclude that "plaquettes" configuration is more favorable.

\section{ELECTRONIC STRUCTURE}

We start from crystal structure of Ba123, which belong to orthorhombic Pnma space group [6]. Main structural motiff of the Ba123 system is a two-leg ladder going along $b$-axis. This ladder is formed by Fe ions which are surrounded by tetrahedrally coordinated Se ions. Those ladders form a kind of "checker board" structure perpendicular to $b$-axis. This structure is obviously quite different from previously studied $\mathrm{BaFe}_{2} \mathrm{As}_{2}$ and $\mathrm{AFe}_{2} \mathrm{Se}_{2}$ systems (see Refs. [5, 10, 11]), which have body centered tetragonal crystal structure.

Using this experimentally established crystal structure (lattice parameters and all atomic positions used in our calculations were taken from Ref. [6]) we performed LDA band structure calculations within the linearized muffin-tin orbitals method (LMTO) [12] using default settings.

In Fig. 1 we present LDA calculated band disper- 
sions (on the right side) and densities of states (DOS) (on the left side). Similarly to Ba122 [10] and $\mathrm{AFe}_{2} \mathrm{Se}_{2}$ [11] systems electronic states on the Fermi level and around it (from 1 to $-2 \mathrm{eV}$ ) are mostly formed by Fe-3d orbitals. The Se- $4 p$ orbitals form bands at energies below $-2 \mathrm{eV}$. Hybridization between Fe-3d and Se-4p states is rather moderate.

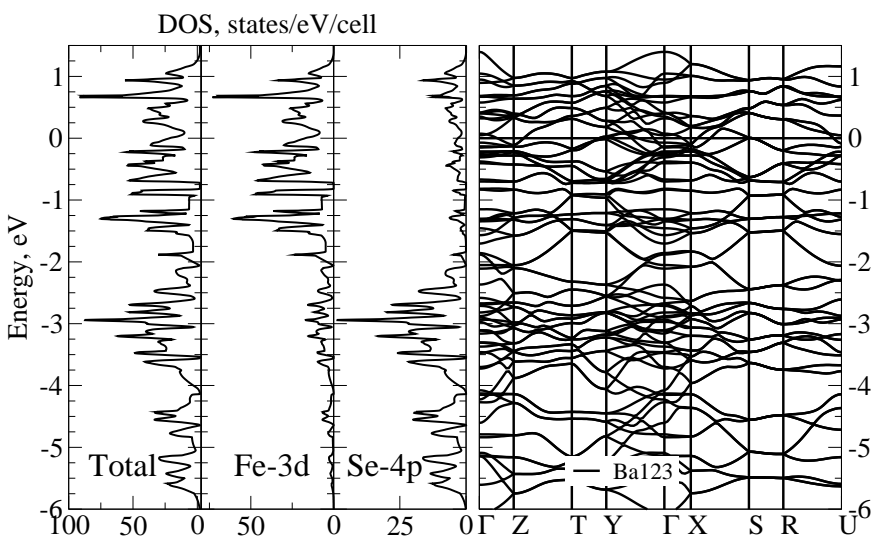

Fig. 1. LDA calculated band dispersions and densities of states of paramagnetic Ba123. The Fermi level $E_{F}$ is at zero energy.

Further on in Fig. 2 we show band dispersions of Ba123 in close proximity of the Fermi level. We can see that these band dispersions are very much different from typical bands of previously studied pnictides or chalcogenides $[10,11]$. Close to $\Gamma$-point there are two electron pockets and near the Brillouin zone border (Ypoint), there are three hole-like pockets (see also Fig. 3, lower panel). Close to the Fermi level there are several band crossings and Van-Hove singularities. Thus Fermi surface topology can be changed rather easy upon doping as in $\mathrm{AFe}_{2} \mathrm{Se}_{2}$ system [11], stressing the potential importance of doping for superconductivity search.

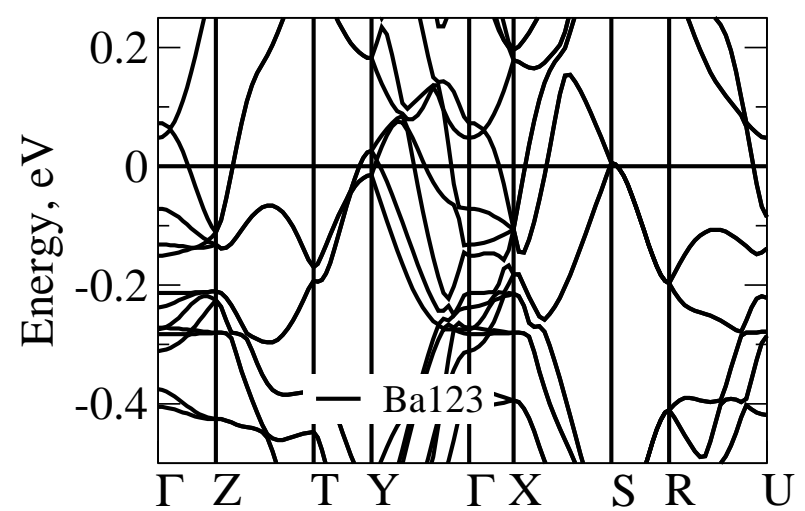

Fig. 2. LDA calculated band dispersions in the vicinity of the Fermi level for Ba123. The Fermi level is at zero energy.
In Fig. 3 we present LDA calculated Fermi surfaces (FS) of Ba123. Overall shape of the Ba123 FS (upper panel of Fig. 4) is very different from those in typical iron pnictides or chalcogenides [10, 11]. First of all it looks pretty three-dimensional and does not have any well established cylinders. Near $\Gamma$-point we see a jabotlike structure and near Y-point we observe a kind of "bottomless pot" FS sheet. In the lower panel of Fig. 3 we show FS cross-section for the $k_{z}=0$ plane. Fermi sheets closest to $\Gamma$-point are electron like, while those FS sheets near Y-point are hole-like.
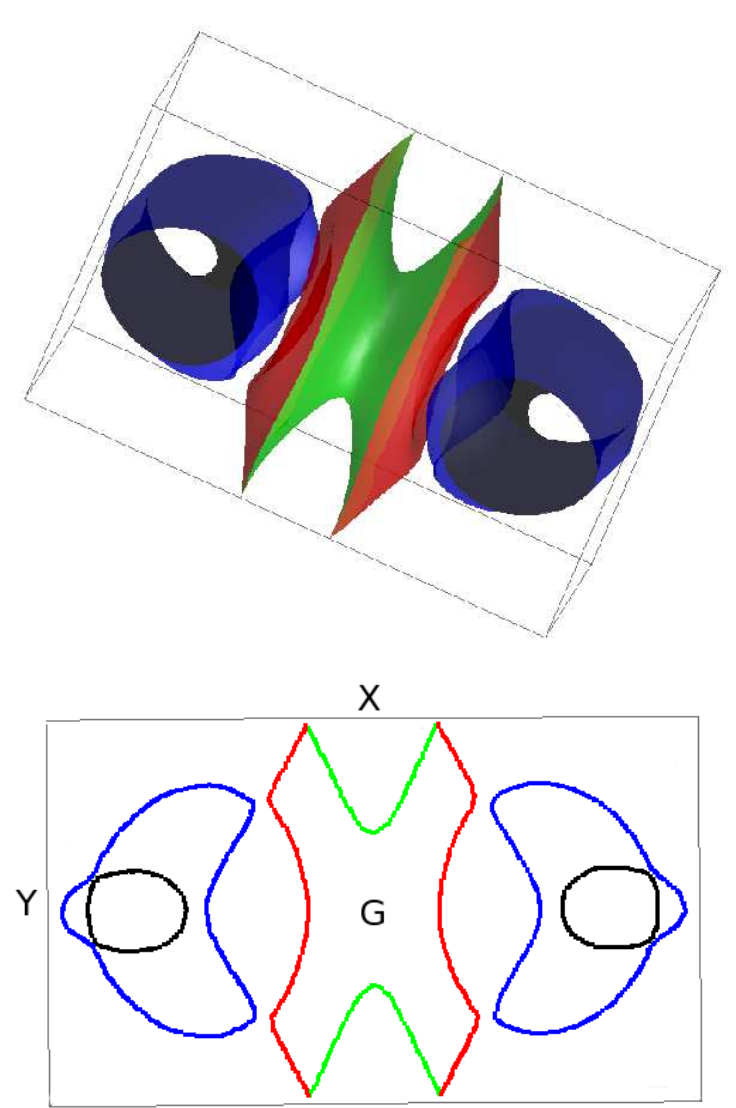

Fig. 3. LDA calculated Fermi surface in the Brillouin zone (upper panel) and $k_{z}=0$ plane Fermi surface crossection (lower panel).

\section{MAGNETIC STRUCTURE}

Neutron scattering experiments $[6,7]$ on magnetic structure of Ba123 at $T \ll T_{N}^{e x p} \sim 250 \mathrm{~K}$ can be interpreted as indicating two possible types of spin configurations, corresponding to irreducible representations $\tau_{1}$ and $\tau_{2}$ of the Pnma space group, giving the same diffraction peaks structure. Since neutron diffraction 
$\tau_{1}$ spin configuration of Ba123 ("plaquettes")

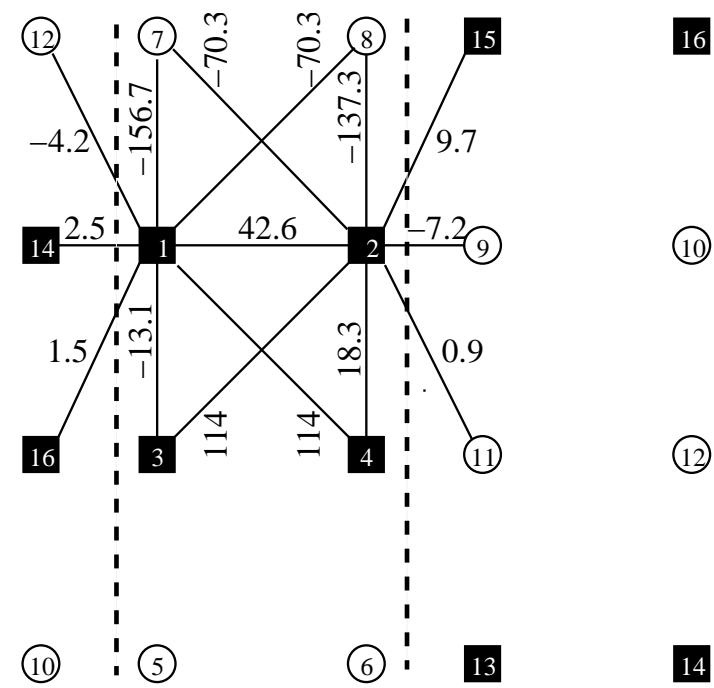

$\tau_{2}$ spin configuration of Ba123 ("zigzags")

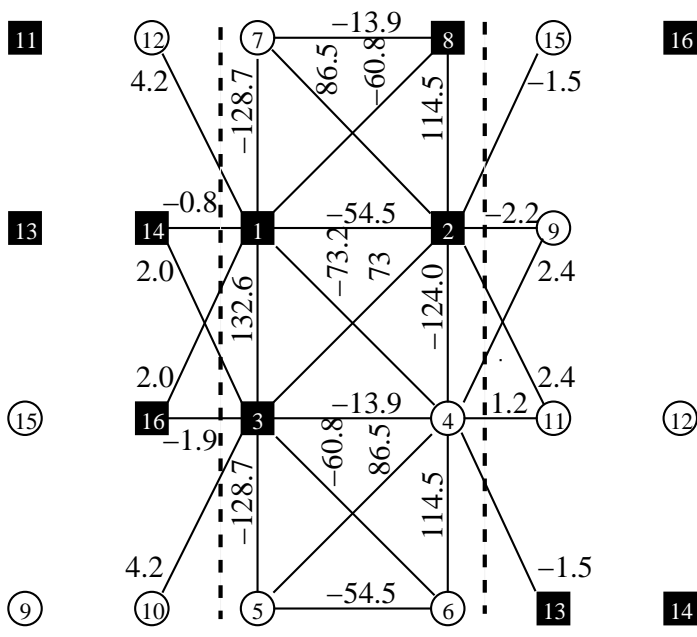

16

\section{1}
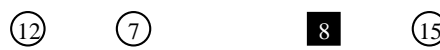

Fig. 4. Experimental variants of AFM spin configurations $\tau_{1}$ (upper panel) and $\tau_{2}$ (lower panel) with LSDA calculated Heisenberg exchange parameter values $(\mathrm{K})$. "Spin up" orientation corresponds to circles and "spin down" to squares. Figures in circles and squares enumerate magnetic sublattuces. Dashed lines separate different ladders.

experiments can not distinguish between these magnetic structures, it is tempting to find more stable configuration theoretically. One can do that by computation of Neel temperature for both configurations within the molecular field approximation, using LSDA calculated Heisenberg exchange interaction parameters.
In case of magnetic structure with several collinear magnetic sublattices Heisenberg model Hamiltonian for classical spin vectors can be written as:

$$
\begin{aligned}
H & =-\frac{1}{2} \sum_{i, n \neq \mathrm{J}, m} I_{i, n, j, m} \vec{S}_{i, n} \vec{S}_{j, m}= \\
& =-\frac{1}{2} \sum_{i, n \neq \mathrm{J}, m} I_{i, n, j, m} S^{2} \vec{e}_{i, n} \vec{e}_{j, m}
\end{aligned}
$$

where $I_{i, n, j, m}$ is exchange integral between site $i$ of sublattice $n$ and site $j$ of sublattice $m, S$ - classical spin vector length and $\vec{e}_{i, n}$ is the unit vector of spin direction. Self-consistent equations for thermodynamic averages of spin $z$-projections $\sigma_{i n} \equiv<S_{i, n}^{z}>$ within mean-field approach can be linearized near Neel temperature $T_{N}$ and written as:

$$
T_{N} \sigma_{i n}=\frac{S^{3}}{3} \sum_{j m} I_{i n, j m} \sigma_{j m}
$$

Due to translation invariance of the crystal the values of $\sigma_{i n}=\sigma_{n}$ are independent of site number in magnetic sublattice. Then Neel temperature $T_{N}$ is determined by the solution of the full system of linear equations for $\sigma_{n}$ for all magnetic sublattices.

The values of exchange integrals for both spin configurations $\left(\tau_{1}\right.$ and $\left.\tau_{2}\right)$ in Ba123 antiferromagnet for classical Heisenberg model at $T=0$ were calculated using the method proposed in Ref. [13]. The results are shown by numbers (in $\mathrm{K}$ ) on bonds in Fig. 4.

Note that both $\tau_{1}$ and $\tau_{2}$ structures can be represented as a set of 16 collinear sublattices with 8 with "spin up" and 8 with "spin down" orientations. The absolute value of $S$ at $T=0$ on each Fe ion in all sublattices is the same. However spins on different sublattices are connected with their magnetic surroundings by different sets of exchange integrals. Thus, at finite temperature the values $\left|\sigma_{n}\right|=\left|<S_{i n}^{2}\right\rangle \mid$ will be different for different sites. We restrict ourselves to the account of only nearest and next nearest exchange bonds of a given ion in the spin-ladder where it belongs and also coupling of nearest neighbors in adjacent ladders.

For $\tau_{1}$ spin structure, using the symmetry of nearest magnetic environment (see Fig. 4, upper panel), we can see that the following relations should be fulfilled:

$$
\begin{aligned}
& \sigma_{1}=\sigma_{3}=-\sigma_{5}=-\sigma_{7}=-\sigma_{10}=-\sigma_{12}=\sigma_{14}=\sigma_{16} \\
& \sigma_{2}=\sigma_{4}=-\sigma_{6}=-\sigma_{8}=-\sigma_{9}=-\sigma_{11}=\sigma_{13}=\sigma_{15}
\end{aligned}
$$

Then the linearized equation for the average value of $z$ projection of $\sigma_{1}$ spin on the magnetic sublattice number 1 becomes: 


$$
\begin{gathered}
T_{N} \sigma_{1}=\frac{S^{3}}{3}\left[J_{1,2}^{0} \sigma_{2}+J_{1,3}^{0} \sigma_{3}+J_{1,7}^{0} \sigma_{7}+\right. \\
\left.+J_{1,4} \sigma_{4}+J_{1,8} \sigma_{8}+2 \tilde{J}_{1,14}^{0} \sigma_{14}+2 \tilde{J}_{1,12} \sigma_{12}+2 \tilde{J}_{1,16} \sigma_{16}\right]
\end{gathered}
$$

where $J_{1,2}^{0}, J_{1,3}^{0}$ and $J_{1,7}^{0}$ are nearest exchange parameters for selected site of the sublattice 1 , with neighbors in 2, 3 and 7 sublattices within the ladder, to which the selected site belongs; $J_{1,4}$ and $J_{1,8}$ are next nearest exchange parameters within the ladder to which the selected site belongs; $\tilde{J}_{1,14}^{0}, \tilde{J}_{1,12}$ and $\tilde{J}_{1,16}$ are adjacent ladder nearest exchange parameters.

Using Eq. (3) the Eq. (4) can be reduced to:

$$
\begin{aligned}
T_{N} \sigma_{1} & =\frac{S^{3}}{3}\left[\left(J_{1,3}^{0}+J_{1,7}^{0}+2 \tilde{J}_{1,14}^{0}-2 \tilde{J}_{1,12}+2 \tilde{J}_{1,16}\right) \sigma_{1}\right. \\
& \left.+\left(J_{1,2}^{0}+J_{1,4}-J_{1,8}\right) \sigma_{2}\right] .
\end{aligned}
$$

Similar equation for $\sigma_{2}$ takes the form:

$$
\begin{aligned}
T_{N} \sigma_{2} & =\frac{S^{3}}{3}\left[\left(J_{2,1}^{0}+J_{2,3}^{0}-J_{2,7}\right) \sigma_{1}+\right. \\
& \left.+\left(J_{2,4}^{0}-J_{2,8}-2 \tilde{J}_{2,9}^{0}+2 \tilde{J}_{2,15}-2 \tilde{J}_{2,11}\right) \sigma_{2}\right]
\end{aligned}
$$

Employing the calculated values of exchange parameters given (in $\mathrm{K}$ ) in Fig. 4, together with calculated value of magnetic moment on iron $\mu_{F e}=2.55 \mu_{B}$ (corresponding classical spin vector value $S=1.275$ ), one can get from Eqs. (5) and (6) the Neel temperature $T_{N}\left(\tau_{1}\right)=217 \mathrm{~K}$, which is quite close to the experimental value of $T_{N}^{e x p} \sim 250 \mathrm{~K}$. Note that the account of exchange bonds with adjacent ladders $\tilde{J}$ only slightly influences the value of $T_{N}$ (without the account of $\tilde{J}$ we get $\left.T_{N}\left(\tau_{1}\right)=204 \mathrm{~K}\right)$. However, the presence of $\tilde{J}$ makes symmetry of exchange surrounding different for sublattices 1 and 2 . This significantly influences the values and signs of exchange bonds along right and left sides of the spin ladder.

Antiferromagnetic structure with spin configuration $\tau_{2}$ is more complicated, however, using magnetic environment symmetry one can establish the following equalities between average values of $z$-projections of spins over all 16 sublattices (see Fig. 4, lower panel).

$$
\begin{gathered}
\sigma_{1}=-\sigma_{5}=-\sigma_{10}=\sigma_{14}, \quad \sigma_{2}=-\sigma_{6}=-\sigma_{9}=\sigma_{13}, \\
\sigma_{3}=-\sigma_{7}=-\sigma_{12}=\sigma_{16}, \quad-\sigma_{4}=\sigma_{8}=\sigma_{11}=-\sigma_{15} .(7)
\end{gathered}
$$

Then following the same line of arguments as for calculation of $T_{N}\left(\tau_{1}\right)$ (Eqs. (4-6)), one can get four equations for $\sigma_{1}, \sigma_{2}, \sigma_{3}$ and $\sigma_{4}$. Again using the calculated value of iron local magnetic moment $\mu_{F e}=2.43 \mu_{B}(S=1.215)$ and exchange integral values (in $\mathrm{K}$ ) (see Fig. 4, lower panel), one can obtain $T_{N}\left(\tau_{2}\right)=186 \mathrm{~K}$. This value is $36 \mathrm{~K}$ lower than $T_{N}\left(\tau_{1}\right)$. This tells us that spin configuration $\tau_{1}$ is, in fact, more favorable. It is probable that one of the reasons for this result is the presence of strong antiferromagnetic exchange $J_{1,2}^{0}=J_{5,6}^{0}=-54 \mathrm{~K}$ in $\tau_{2}$ structure.

\section{CONCLUSION}

In this work we have studied the band structure, density of states and Fermi surfaces of recently discovered possible Fe-based superconductor $\mathrm{BaFe}_{2} \mathrm{Se}_{3}$ (Ba123). In contrast to typical representatives of iron pnictide and chalcogenide systems $[10,11]$ and despite rather similar chemical composition, Ba123 has completely different crystal structure. Instead of planes with square lattice of Fe-ions, Ba123 is a spin-ladder system with two-leg ladders of Fe ions, which form checkerboard pattern in the plane perpendicular to the ladder direction. Thus, not unexpectedly, the band dispersions and Fermi surfaces are quite different from those in $\mathrm{BaFe}_{2} \mathrm{As}_{2}$ and (K,Cs)Fe $\mathrm{Se}_{2}$. Complicated band structure of Ba123 in the vicinity of the Fermi level illustrates the importance of experiments on different kinds of doping in search for possible superconductivity in this system.

For the undoped Ba123 spin-ladder system, there is a problem of correct choice among two possible antiferromagnetic spin configurations, not easily discernible by neutron scattering ("plaquettes" versus "zigzags"). We could distinguish these configurations by calculating the Neel temperature for classical Heisenberg model, within the molecular field approximation. Corresponding Heisenberg exchange integral values were obtained from LSDA calculations. From our estimates we conclude that more favorable is "plaquette" configuration with (calculated) $T_{N}\left(\tau_{1}\right)=217 \mathrm{~K}$ (in contrast to "zigzags" with calculated $\left.T_{N}\left(\tau_{2}\right)=186 \mathrm{~K}\right)$. This conclusion agrees with experimental evidence of Ref. [7] and total LSDA energy calculations of Ref. [9].

This work is partly supported by RFBR grant 1102-00147 and was performed within the framework of the Program of fundamental research of the Russian Academy of Sciences (RAS) "Quantum mesoscopic and disordered structures" (12-П-2-1002).

1. Y. Kamihara, T. Watanabe, M. Hirano, H. Hosono. J. Am. Chem. Soc. 130, 3296-3297 (2008).

2. M.V. Sadovskii, Uspekhi Fiz. Nauk 178, 1243 (2008); Physics Uspekhi 51, No. 12 (2008); arXiv: 0812.0302.

3. K. Ishida, Y. Nakai, H. Hosono. J.Phys. Soc. Jpn. 78, 062001 (2009).

4. Y. Mizuguchi, Y. Takano. J. Phys. Soc. Jpn. 79, 102001 (2010). 
5. M.V. Sadovskii, E.Z. Kuchinskii, I.A. Nekrasov, arXiv:1106.3707.

6. A. Krzton-Maziopa, E. Pomjakushina, V. Pomjakushin, D. Sheptyakov, D. Chernyshov, V. Svitlyk, K. Conder, J.Phys.:Condens.Matter 23, 402201 (2011).

7. J. M. Caron, J. R. Neilson, D. C. Miller, A. Llobet, T. M. McQueen, arXiv:1108.2928.

8. H. Lei, H. Ryu, A. Frenkel, C. Petrovic, arXiv:1110.3039.

9. B. Saparov, S. Calder, B. Sipos, H. Cao, S. Chi, D. J. Singh, A. D. Christianson, M. D. Lumsden, A. S. Sefat, arXiv:1110.2439.

10. I.A. Nekrasov, Z.V. Pchelkina, M.V. Sadovskii. Pis'ma Zh. Eksp. Teor. Fiz. 88, 155 (2008) [JETP Letters 88, 144 (2008)], arXiv:0806.2630.

11. I.A. Nekrasov, M.V. Sadovskii Pis'ma Zh. Eksp. Teor. Fiz. 93, 182 (2011) [JETP Letters 93, 166 (2011)], arXiv:1101.0051.

12. O.K. Andersen. Phys. Rev. B 12, 3060 (1975); O. Gunnarsson, O. Jepsen, O.K. Andersen. Phys. Rev. B 27, 7144 (1983); O.K. Andersen, O. Jepsen. Phys. Rev. Lett. 53, 2571 (1984).

13. A. I. Liechtenstein, M. I. Katsnelson, V. P. Antropov, V. A. Gubanov, J. Magn. Magn. Mater. 67, 65 (1987); V. I. Anisimov, F. Aryasetiawan, A. I. Lichtenstein, J. Phys.: Condens. Matter 9, 767 (1997). 